\title{
Nail Discoloration, CTCAE
}

National Cancer Institute

\section{Source}

National Cancer Institute. Nail Discoloration, CTCAE. NCI Thesaurus. Code C143694.

A disorder characterized by a change in the color of the nail plate. 